\title{
Hydroxyzine may be safe and effective in generalised anxiety disorder
}

\author{
Llorca PM, Spadone C, Sol O et al. Efficacy and safety of hydroxyzine in the treatment of generalized anxiety disorder: a \\ 3-month double-blind study.J Clin Psychiatry 2002 Nov;63:1020-7.
}

\section{QUESTION: Is hydroxyzine safe and effective for generalised anxiety disorder in adult outpatients?}

\section{Design}

Randomised trial. Investigators and participants were blind to treatment allocation.

\section{Setting}

89 French general practices (supervised by psychiatrists); January 1998-March 1999.

\section{Participants}

334 outpatients diagnosed with generalised anxiety disorder (DSM-IV criteria) and a Hamilton Rating Scale for Anxiety (HAM-A) total score of at least 20. All were aged 18-65 years (mean 45 years); 68\% women. Exclusion criteria were alcohol or drug dependence; major depressive episode within 6 months; score of 7 or above on the Raskin Severity of Depression and Mania scale; psychotic or delusional disorders within 3 years; chronic disease; prostate cancer; closed angle glaucoma; antidepressants, neuroleptics, mood regulators, morphine or derivatives, hydroxyzine, or bromazepam within 4 weeks; benzodiazepines more than 2 days per week during the past 30 days; benzodiazepines during the previous 2 weeks; need for psychotherapy; contraindication to study medication; and women who were pregnant, breastfeeding or not using contraception.

\section{Intervention}

After a 2 week placebo run in phase, participants received 12 weeks of $50 \mathrm{mg}$ hydroxyzine per day, $6 \mathrm{mg}$ bromazepam per day or placebo. Treatment was divided into three daily doses. Treatment was followed by 4 weeks of run out placebo. $83 \%$ of participants completed treatment. Adherence to medication was $85 \%$.

\section{Main outcome measures}

Response (mean change in HAM-A total score); remission; adverse effects.

\section{Main results}

Compared with placebo, after 3 months of treatment the hydroxyzine group had superior response rates $(\mathrm{P}=0.003)$; remission rates $(\mathrm{P}=0.028)$; changes in Clinical Impressions Severity score $(\mathrm{P}=0.001)$; maintenance of efficacy ( $\mathrm{P}=0.022)$, and Hospital Anxiety and Depression scale score on day $84(\mathrm{P}=0.008)$. There was no significant difference in efficacy between hydroxyzine and bromazepam. Safety was comparable between groups. Drowsiness was the most commonly reported adverse effect in the hydroxyzine group, although drowsiness was twice as common with bromazepam (see table).

\section{Conclusions}

Hydroxyzine appears to be safe and effective in adults with generalised anxiety disorder.

\section{COMMENTARY}

Generalised Anxiety Disorder (GAD) is the most common anxiety disorder, although it is generally less severe than panic disorder. People with GAD often present to general practitioners. The most common pharmacological treatments are benzodiazepines, buspirone and venlafaxine, a dual noradrenergic-serotonergic reuptake inhibitor. ${ }^{1}$

Although benzodiazepines provide short term relief, they may not be a good long term treatment given the chronic, non-remitting, relapsing nature of GAD. Benzodiazepines may be associated with cognitive impairment, interact with CNS depressants and have substantial potential for abuse, tolerance, dependence and withdrawal effects. ${ }^{2}$ These difficulties suggest the need for alternative medications.

In recent years there has been growing interest in the psychiatric effects of non-psychiatric drugs. Llorca et al found that hydroxyzine is an effective anxiolytic in outpatients with GAD. Moreover, it did not produce symptoms of withdrawal on discontinuation and it tended to be well tolerated. The study is well designed and supports previous findings. As the authors point out, however, the study was not designed to compare the efficacy and onset of action of hydroxyzine versus bromazepam. Before being applied in widespread practice, additional studies are needed to compare hydroxyzine with high potency benzodiazepines.

Professor Associate Shahin Akhondzadeh, PhD Roozbeh Psychiatric Hospital Tehran University of Medical Sciences Tehran, Iran

1 Bruce Lydiard R. An overview of generalized anxiety disorder: disease state- appropriate therapy. Clin Ther 2000; 22: A3-24.

2 Akhondzadeh S, Naghavi HR, Vazirian M et al. Passionflower in the treatment of generalized anxiety: A double-blind and randomized trial with oxazepam.J Clin Pharm E Ther 2001; 26: 363-7.

Adverse effects in adults with generalised anxiety disorder receiving hydroxyzine, bromazepam or placebo

\begin{tabular}{lcc} 
Adverse effect & Hydroxyzine \%(n=102) & Bromazepam \%(n=114) \\
Experienced at least 1 adverse event & 41 & 45 \\
\hline Experienced at least 1 adverse event due to treatment & 15 & 45 \\
\hline Drowsiness & 4 & 14 \\
\hline
\end{tabular}

Note: Drowsiness was the only significant different between groups: bromazepam $>$ hydroxyzine and placebo $(p<0.05)$. 